\title{
Artificial Immune Systems Applied in Data Management Solutions to the Problem of Restoration of Electrical Distribution Systems
}

\author{
A. M. Cossi and M. L. M. Lopes
}

\begin{abstract}
This paper proposes a method based on Artificial Immune Systems for data management solutions provided by a model used to train power grid and find possible solutions to the problem of restoration of electricity distribution systems. The method aims to manage the supply of recurring defects solutions in the power grid through information stored in a database; the network training for non-recurring defects and storage of data in the database; choosing solutions in the database to start the network training process in the search for new solutions. To train the network and find new solutions to the restoration problem, we use a model that has as one solution technique Tabu Search algorithm. To test the efficiency of the proposed method, we present results of tests and discussions carried out in a medium voltage distribution system.
\end{abstract}

Keywords - Data management, artificial immune systems, distribution systems, restoration.

\section{INTRODUÇÃO}

A S FERRAMENTAS desenvolvidas para resolver o Problema de Restauração de Sistemas de Distribuição de Energia Elétrica (PRSDEE) devem ser eficientes para proporcionar uma resposta rápida para a restauração do sistema evitando assim que as empresas distribuidoras de energia elétrica deixem de cumprir com as metas de qualidade impostas pelos órgãos reguladores. Neste contexto, este trabalho busca contribuir com essas ferramentas através de um método baseado em Sistemas Imunológicos Artificiais (SIA), cujo objetivo é de gerenciar os dados de soluções de PRSDEE's e tentar promover um restabelecimento do sistema de forma mais rápida.

O método proposto consiste em gerenciar: 1- o fornecimento de soluções de PRSDEE's recorrentes através de informações armazenadas na memória de um banco de dados; 2- o treinamento da rede para PRSDEE's não recorrentes e memorização dos dados das soluções na memória do banco de dados; 3- o fornecimento de soluções existentes na memória do banco de dados que sejam de boa qualidade para iniciar processos de treinamento de redes elétricas na busca de novas soluções para resolver o PRSDEE. O fato de iniciar o processo de treinamento da rede elétrica na busca de soluções de PRSDEE's através de uma solução de boa qualidade, pode reduzir significativamente o espaço de busca de soluções e consequentemente baixar o tempo de processamento, gerando assim uma resposta mais rápida para o PRSDEE.

Este trabalho contou com o apoio financeiro da FAPESP processo 13/02348-4.

A. M. Cossi, Universidade Estadual Paulista "Júlio de Mesquita Filho" (UNESP), Ilha Solteira, São Paulo, Brasil, cossi@mat.feis.unesp.br

M. L. M. Lopes, Universidade Estadual Paulista "Júlio de Mesquita Filho" (UNESP), Ilha Solteira, São Paulo, Brasil, mara@mat.feis.unesp.br

Corresponding author: Antonio Marcos Cossi
O método baseado em SIA funciona através do reconhecimento de cadeias de padrões pré-determinadas pelo sistema imunológico entre as células com anomalias e suas respectivas cura. Neste caso, para cada anomalia ocorrida na célula, a qual é identificada através de um antígeno, é verificada a existência ou não de anticorpos (cura) para o antígeno apresentado pela anomalia nessa célula. Assim, o método poderá fornecer anticorpos para anomalias já conhecidas em determinadas células ou criar anticorpos para anomalias desconhecidas. O funcionamento da técnica SIA é detalhada em [1] e [2].

$\mathrm{Na}$ literatura especializada existem diversos trabalhos que abordam o tema sobre PRSDEE's. Dentre eles podemos destacar os que utilizam como técnica de solução os métodos heurísticos [3] e metaheurísticos: algoritmos genéticos [4] e [5], tabu search [6], sistemas imunológicos artificiais [7], dentre outros. No entanto, não existem trabalhos que tratam especificamente do problema de gerenciamento de dados de soluções de problemas ligados a sistemas de distribuição de energia elétrica utilizando SIA.

$\mathrm{O}$ método proposto pode ser utilizado para treinar a rede considerando diversas situações de PRSDEE's e armazenar as soluções na memória do banco de dados para que possam ser utilizadas em casos de defeitos na rede de distribuição, obtendo assim uma resposta rápida para o PRSDEE. Além disso, o método pode ser adaptado e utilizado em conjunto com outras ferramentas utilizadas para resolver problemas diversos ligados a sistemas de distribuição de energia elétrica. Para treinar a rede de distribuição na busca de soluções e resolver o PRSDEE utiliza-se um modelo de restauração que possui como técnica de solução um algoritmo tabu search (TS). Ressalta-se que não é objeto deste trabalho apresentar o modelo de treinamento da rede elétrica utilizando o algoritmo TS.

Para testar a eficiência do método proposto de gerenciamento de dados de soluções para o PRSDEE, apresentam-se resultados e discussões de testes realizados em um sistema de distribuição de média tensão, cujos dados podem ser encontrados em [8].

O trabalho está organizado da seguinte forma: na Seção 2 apresenta-se em detalhes o método proposto baseado em SIA; na Seção 3 apresentam-se os resultados e comentários dos testes; e na Seção 4 as conclusões finais.

\section{MÉTOdo BASEAdo EM SIA}

O método baseado em SIA desenvolvido para o gerenciamento de dados é chamado de MGD-SIA (Método de Gerenciamento de Dados Baseado em Sistemas Imunológicos Artificiais). No PRSDEE o MGD-SIA identifica um sistema de distribuição como sendo uma célula. Um defeito no sistema 
de distribuição é então conhecido como uma anomalia nessa célula. Uma anomalia na célula é então identificada pelo defeito ocorrido em uma determinada seção do sistema de distribuição, a qual é reconhecido pelo MGD-SIA através de um agente patológico chamado de antígeno (identificação da seção onde o defeito ocorreu). Uma possível solução para o defeito ocorrido no sistema de distribuição (anomalia na célula) é reconhecida pelo MGD-SIA como sendo um anticorpo com afinidade total ou parcial ao antígeno da anomalia. Tal anticorpo seria então uma solução para o problema da restauração total ou parcial do sistema de distribuição após a ocorrência do defeito, ou seja, seria a cura para a anomalia na célula. A Fig. 1 ilustra de forma geral o método proposto.

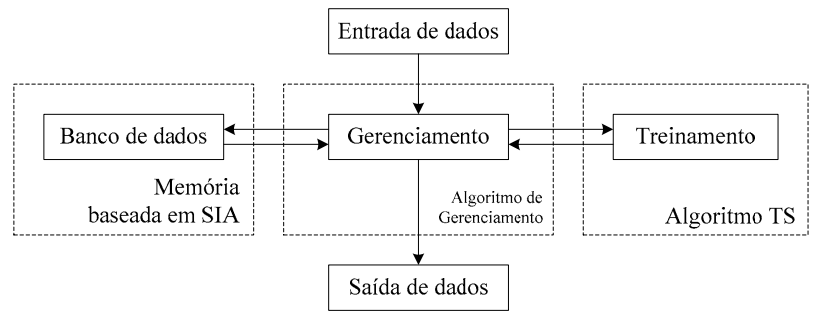

Figura. 1. Esquema geral do método baseado em SIA (MGD-SIA).

De acordo com a Fig. 1, temos:

- Entrada de dados: são os dados da célula e da anomalia (antígeno) ocorrida na célula, ou seja, são os dados do sistema elétrico, do defeito e das seções da rede afetadas pelo defeito;

- Gerenciamento: trata-se de um algoritmo de gerenciamento de dados. Este algoritmo realiza todo o processo de análise, comparação e escolha dos dados, bem como a comunicação entre a entrada de dados, o banco de dados, o treinamento e a saída de dados;

- Treinamento: algoritmo TS desenvolvido para avaliar o defeito e encontrar possíveis curas (anticorpos) para anomalias desconhecidas (antígeno) ocorrida na célula, ou seja, algoritmo TS desenvolvido para encontrar as possíveis soluções para o PRSDEE;

- Banco de dados: armazena os dados de células que já foram analisadas e de seus respectivos antígenos e anticorpos através de cadeias de padrões e suas respectivas topologias de rede, ou seja, armazena dados de sistemas elétricos que já foram estudados e dos defeitos e suas respectivas soluções.

- Saída de dados: são os padrões de anticorpos para o antígeno e suas respectivas topologias de rede, ou seja, são as possíveis soluções para o PRSDEE.

A seguir, apresenta-se de forma detalhada o MGD-SIA.

\subsection{Algoritmo de Gerenciamento do MGD-SIA}

$\mathrm{O}$ algoritmo de gerenciamento do MGD-SIA funciona da seguinte maneira:

1. Detecção de anomalia na célula;

2. Identificação, localização e classificação do agente patológico (antígeno);
3. Verificar se a célula já foi analisada e se o antígeno é conhecido:

3.1. Se a célula nunca foi analisada, ir ao passo 4.1 (resposta primária).

3.2. Se a célula já foi analisada e o antígeno não for conhecido, ir ao passo 4.2 (resposta primária).

3.3. Se a célula já foi analisada e o antígeno for conhecido, ir ao passo 5 (resposta secundária).

4. Resposta primária:

4.1. Se a célula nunca foi analisada, encontrar a cura através da produção de anticorpos com afinidade total ou parcial ao antígeno através do treinamento da rede utilizando o algoritmo TS, da seguinte maneira:

4.1.1. Guardar a nova célula no conjunto de células analisadas;

4.1.2. Guardar o novo antígeno no conjunto de antígenos ligados à célula;

4.1.3. Produzir padrões de anticorpos através do treinamento da rede sem utilizar uma solução inicial conhecida;

4.1.4. Escolher o anticorpo com maior afinidade ao antígeno (melhor função de adaptação);

4.1.5. Guardar o anticorpo no conjunto de anticorpos ligados à célula;

4.1.6. Adotar a cadeia de padrões do anticorpo como a cura para a anomalia;

4.1.7. Ir ao passo 6 .

4.2. Se a célula já foi analisada, porém o antígeno não é conhecido, encontrar a cura através da produção de anticorpos com afinidade total ou parcial ao antígeno através do treinamento da rede utilizando o algoritmo TS, da seguinte maneira:

4.2.1. Guardar o novo antígeno no conjunto de antígenos ligados à célula;

4.2.2. Verificar a possibilidade de iniciar $\mathrm{o}$ treinamento da rede através de algum anticorpo, pertencente ao conjunto de anticorpos, com alguma afinidade parcial ao antígeno (iniciar o treinamento da rede através de uma solução inicial de boa qualidade), da seguinte maneira:

$i$. Calcular a taxa de afinidade $T_{A f}$ do antígeno;

ii. Apresentar o antígeno ao conjunto de anticorpos. Neste caso calcula-se a afinidade $A_{f t}$ de cada anticorpo em relação ao antígeno;

iii. Se um ou mais anticorpos apresentarem uma $A_{f t}$ maior que $T_{A f}$, este(s) anticorpo(s) possui(em) alguma afinidade ao antígeno. Neste caso, o anticorpo que possuir a maior afinidade será a solução inicial do algoritmo TS. Ir ao passo 4.2.3. Caso contrário, ir ao passo 4.2.4 e iniciar o treinamento da rede normalmente (sem solução inicial previamente conhecida).

4.2.3. Produzir anticorpos através do treinamento da rede utilizando uma solução inicial conhecida e escolher aquele com maior afinidade ao 
antígeno (melhor função de adaptação). Após, ir ao passo 4.2.5.

4.2.4. Produzir anticorpos através do treinamento da rede sem utilizar uma solução inicial conhecida e escolher aquele com maior afinidade ao antígeno (melhor função de adaptação). Após, ir ao passo 4.2.5.

4.2.5. Guardar o anticorpo no conjunto de anticorpos ligados à célula;

4.2.6. Adotar a cadeia de padrões do anticorpo como a cura para a anomalia;

4.2.7. Ir ao passo 6 .

5. Resposta secundária: se a célula e o antígeno forem conhecidos, encontrar a cura através de um anticorpo com afinidade total ou parcial ao antígeno, da seguinte maneira:

5.1. Apresentar o antígeno a todos os anticorpos do conjunto de anticorpos;

5.2. Verificar qual é o anticorpo correspondente ao antígeno (mesma cadeia de padrões);

5.3. Apresentar a cadeia de padrões do anticorpo como cura para a anomalia;

5.4. Ir ao passo 6.

6. Gerar a saída de dados, podendo ser uma das soluções:

6.1. Solução 1: cadeia de padrões do anticorpo com maior afinidade (total ou parcial) ao antígeno gerado pela resposta primária;

6.2. Solução 2: cadeia de padrões do anticorpo com maior afinidade (total ou parcial) ao antígeno gerado pela resposta secundária;

6.3. Solução 3: nenhum anticorpo. Neste caso não foi encontrada nenhuma cura para a anomalia na célula. O sistema não será restaurado.

A Fig. 2 ilustra de forma resumida o algoritmo de gerenciamento do MGD-SIA descrito anteriormente.

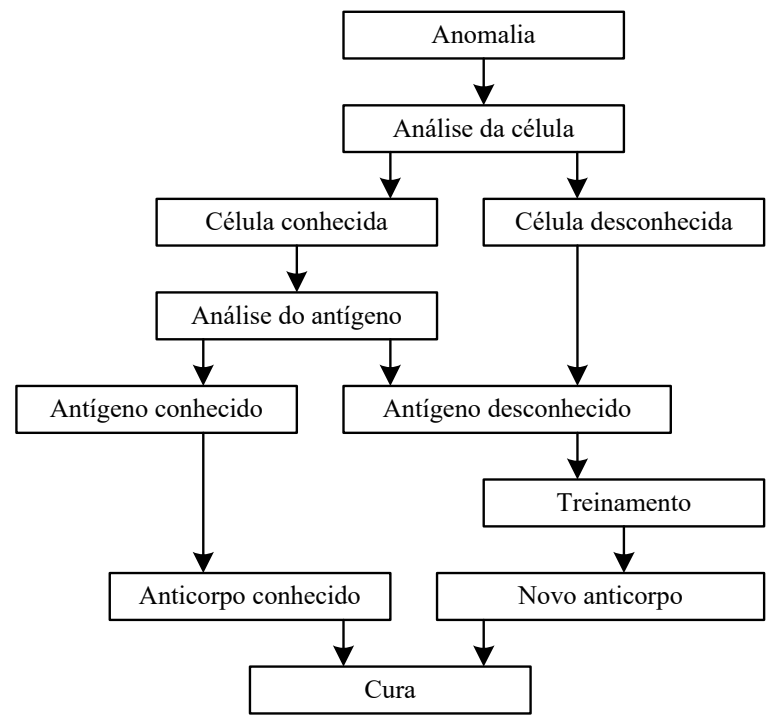

Figura. 2. Resumo do algoritmo de gerenciamento do MGD-SIA.

\subsection{Memória do MGD-SIA}

$\mathrm{O}$ esquema de memória do MGD-SIA consiste em armazenar dados de células estudadas após a ocorrência de uma anomalia nessa célula. Neste caso, são armazenados na memória do banco de dados o conjunto de células e os seus respectivos padrões de antígenos (dados da rede elétrica com defeito) e anticorpos (dados da rede elétrica após o restabelecimento da rede), bem como todos os demais dados da rede elétrica. A busca por soluções na memória do banco de dados consiste em analisar a afinidade entre os padrões de antígeno e anticorpos. Esse esquema de memória é detalhado a seguir.

\section{A. Conjunto de células conhecidas (LC)}

A célula representa um sistema elétrico, e é conhecida por um nome de identificação conforme a quantidade de seções. Cada seção é considerada como sendo uma região da rede de distribuição composta por várias barras de carga (consumidores).

O conjunto de células LC é formado por células conhecidas:

- $\mathrm{LC}=\left\{\mathrm{C}_{12}, \mathrm{C}_{34}, \mathrm{C}_{120}, \ldots, \mathrm{C}_{\mathrm{ns}}\right\}$

sendo: $\mathrm{C}_{12}$ é o sistema $\mathrm{S} 12$ (sistema de 12 seções); $\mathrm{C}_{34}$ é o sistema S34 (sistema de 34 seções); $\mathrm{C}_{120}$ é o sistema S120 (sistema de 120 seções), $\mathrm{C}_{\mathrm{ns}}$ é o sistema $\mathrm{S}_{\mathrm{ns}}$ (sistema de $n s$ seções).

\section{B. Conjunto de antígenos ligados à célula (LAg)}

Um antígeno representa a anomalia (agente patológico) na célula. Assim, um defeito na seção $k$ da célula $\mathrm{C}_{\mathrm{j}}$ (rede elétrica) teria o antígeno $\mathrm{AgC}_{\mathrm{j}}^{\mathrm{k}}$. Portanto, o antígeno é identificado pelo número da seção em que a anomalia acorreu na célula. Outro defeito em outra seção da célula seria outro antígeno ligado à mesma célula e assim por diante.

$\mathrm{O}$ conjunto de antígenos $\mathrm{LAgC}_{\mathrm{j}}$ é formado pelos antígenos ligados a célula sob análise:

- $\mathrm{LAgC}_{\mathrm{j}}=\left\{\mathrm{AgC}_{\mathrm{j}}^{1}, \mathrm{AgC}_{\mathrm{j}}^{2}, \ldots, \mathrm{AgC}_{\mathrm{j}}^{\mathrm{k}}, \ldots, \mathrm{AgC}_{\mathrm{j}}^{\mathrm{n}}\right\}$

- $\quad \mathrm{AgC}_{\mathrm{j}}^{\mathrm{k}}=\left\{\mathrm{S}_{1} \mathrm{C}_{\mathrm{j}}^{\mathrm{k}}, \mathrm{S} 2 \mathrm{C}_{\mathrm{j}}^{\mathrm{k}}, \ldots, \mathrm{SiC}_{\mathrm{j}}^{\mathrm{k}}, \ldots, \mathrm{SnC}_{\mathrm{j}}^{\mathrm{k}}\right\}$

sendo: $\mathrm{LAgC}_{\mathrm{j}}$ é o conjunto formado por diferentes antígenos representando diferentes anomalias ocorridas na célula $C_{j}$; $\mathrm{AgC}_{\mathrm{j}}^{\mathrm{k}}$ é o antígeno que representa a anomalia ocorrida na seção $k$ da célula $C_{\mathrm{j}}$, e é formado por uma cadeia de padrões composto por: seção do defeito (seção da anomalia - $\mathrm{S}_{\mathrm{a}}$ ), seções que foram desenergizadas pelo defeito (seções com padrões não próprios - $\mathrm{S}_{\mathrm{np}}$ ) e seções que não foram desenergizadas (seções com padrões próprios $-\mathrm{S}_{\mathrm{p}}$ ); $\mathrm{SiC}_{\mathrm{j}}^{\mathrm{k}}$ é a seção $i$ da cadeia de padrões do antígeno $\mathrm{AgC}_{\mathrm{j}}^{\mathrm{k}}$.

\section{Conjunto de anticorpos ligados à célula conhecida (LAb)}

Um anticorpo representa a cura para a anomalia na célula. $\mathrm{O}$ anticorpo deve ter afinidade total ou parcial ao antígeno. Assim, um defeito na seção $k$ da célula $C_{j}$ teria o antígeno $\mathrm{AgC}_{\mathrm{j}}^{\mathrm{k}}$. Portanto, o anticorpo com afinidade ao antígeno $\mathrm{AgC}_{\mathrm{j}}^{\mathrm{k}}$ seria $\mathrm{AbC}_{\mathrm{j}}^{\mathrm{k}}$. 
$\mathrm{O}$ conjunto de anticorpos $\mathrm{LAbC}_{\mathrm{j}}$ é formado pelos anticorpos com alguma afinidade a seus respectivos antígenos ligados a célula sob análise:

- $\mathrm{LAbC}_{\mathrm{j}}=\left\{\mathrm{AbC}_{\mathrm{j}}^{1}, \mathrm{AbC}_{\mathrm{j}}^{2}, \ldots, \mathrm{AbC}_{\mathrm{j}}^{\mathrm{k}}, \ldots, \mathrm{AbC}_{\mathrm{j}}^{\mathrm{n}}\right\}$

- $\mathrm{AbC}_{\mathrm{j}}^{\mathrm{k}}=\left\{\mathrm{S}_{1} \mathrm{C}_{\mathrm{j}}^{\mathrm{k}}, \mathrm{S} 2 \mathrm{C}_{\mathrm{j}}^{\mathrm{k}}, \ldots, \mathrm{SiC}_{\mathrm{j}}^{\mathrm{k}}, \ldots, \mathrm{SnC}_{\mathrm{j}}^{\mathrm{k}}\right\}$

sendo: $\mathrm{LAbC}_{\mathrm{j}}$ é o conjunto de anticorpos com alguma afinidade aos seus respectivos antígenos para diferentes anomalias ocorridas célula $\mathrm{C}_{\mathrm{j}} ; \mathrm{AbC}_{\mathrm{j}}^{\mathrm{k}}$ é o anticorpo com afinidade ao antígeno da anomalia ocorrida na seção $k$ da célula $C_{j}$, e é representado por uma cadeia de padrões composta pelas seções $S_{a}, S_{n p}$ (seções restauradas ou não pelo algoritmo TS) e $\mathrm{S}_{\mathrm{p}}$; $\mathrm{SiC}_{\mathrm{j}}^{\mathrm{k}}$ é uma seção $i$ da cadeia de padrões do anticorpo $\mathrm{AbC}_{\mathrm{j}}^{\mathrm{k}}$.

Se a cadeia de padrões do anticorpo $\mathrm{AgC}_{\mathrm{j}}^{\mathrm{k}}$ apresentar $\mathrm{biC}_{\mathrm{j}}^{\mathrm{k}}=0$ na posição correspondente da seção $i$ ou para qualquer seção do anticorpo, significa que esta seção não foi religada pelo processo de restauração.

\subsection{Cadeia de padrões}

As Fig. 3 e 4 ilustram o casamento entre as cadeias de padrões de antígeno e anticorpo. Os vetores são formados pelas seções $S_{a}, S_{n p}$ e $S_{p}$, e pelo vetor afinidade entre essas cadeias.

No MGD-SIA, os padrões (antígeno e anticorpo) a serem analisados são os padrões próprios e não-próprios. Neste caso, a seção do defeito referente ao antígeno analisado não entra na análise de afinidade entre as cadeias, apesar de fazer parte da cadeia de padrões.

\section{A. Padrões com afinidade total}

Para haver afinidade total entre os padrões analisados do anticorpo e antígeno todas as posições do vetor afinidade deve possuir 1 (um). Neste caso, para o antígeno $\mathrm{AgC}_{\mathrm{j}}^{\mathrm{k}}$ existe um anticorpo $\mathrm{AbC}_{\mathrm{j}}^{\mathrm{k}}$, ou seja, existe o casamento total entre as cadeias de padrões do antígeno e anticorpo. A Fig. 3 ilustra um exemplo de afinidade total do antígeno $\mathrm{AgC}_{\mathrm{j}}^{\mathrm{k}}$ com o anticorpo $\mathrm{AgC}_{\mathrm{j}}^{\mathrm{k}}$.

\begin{tabular}{|c|c|c|c|c|c|c|}
\hline $\mathrm{S}_{\mathrm{a}}$ & $S_{p}$ & $\mathrm{~S}_{\mathrm{np}}$ & $\mathrm{S}_{\mathrm{np}}$ & & $\mathrm{S}_{\mathrm{p}}$ & \\
\hline $\mathrm{b}_{\mathrm{i}} \mathrm{C}_{\mathrm{j}}^{\mathrm{k}}$ & $\mathrm{b}_{2} \mathrm{C}_{\mathrm{j}}^{\mathrm{k}}$ & $\mathrm{b}_{3} \mathrm{C}_{\mathrm{j}}^{\mathrm{k}}$ & $\mathrm{b}_{4} \mathrm{C}_{\mathrm{j}}^{\mathrm{k}}$ & $\ldots$ & $\mathrm{b}_{\mathrm{n}} \mathrm{C}_{\mathrm{j}}^{\mathrm{k}}$ & $\mathrm{AgC}_{\mathrm{j}}^{\mathrm{k}}$ \\
\hline$b_{i} C_{j}^{k}$ & $\mathrm{~b}_{2} \mathrm{C}_{\mathrm{j}}^{\mathrm{k}}$ & $\mathrm{b}_{3} \mathrm{C}_{\mathrm{j}}^{\mathrm{k}}$ & $\mathrm{b}_{4} \mathrm{C}_{\mathrm{j}}^{\mathrm{k}}$ & $\ldots$ & $\mathrm{b}_{\mathrm{n}} \mathrm{C}_{\mathrm{j}}^{\mathrm{k}}$ & $A b C_{j}^{k}$ \\
\hline - & 1 & 1 & 1 & $\cdots$ & 1 & Afinic \\
\hline
\end{tabular}

Figura 3. Comparação entre cadeia de padrões de antígeno e anticorpo com afinidade total.

\section{B. Padrões com afinidade parcial}

Caso alguma posição do vetor afinidade possua o valor 0 (zero), significa que não existe afinidade total do anticorpo ao antígeno, ou seja, para o antígeno $\mathrm{AgC}_{\mathrm{j}}^{\mathrm{k}}$ não existe um anticorpo $\mathrm{AbC}_{\mathrm{j}}^{\mathrm{k}}$ com afinidade total. Neste caso, poderá haver ou não um anticorpo uma afinidade parcial ao antígeno. A Fig. 4 ilustra um exemplo de afinidade parcial do antígeno $\mathrm{AgC}_{\mathrm{j}}^{\mathrm{k}}$ com algum anticorpo da lista de anticorpos, como por exemplo o anticorpo $\mathrm{AgC}_{\mathrm{j}}^{\mathrm{g}}$.

\begin{tabular}{|c|c|c|c|c|c|c|}
\hline $\mathrm{S}_{\mathrm{a}}$ & $\mathrm{S}_{\mathrm{p}}$ & $S_{n p}$ & $\mathrm{~S}_{\mathrm{np}}$ & & $\mathrm{S}_{\mathrm{p}}$ & \multirow[b]{2}{*}{$\mathrm{AgC}_{j}^{k}$} \\
\hline $\mathrm{b}_{\mathrm{i}} \mathrm{C}_{\mathrm{j}}^{\mathrm{k}}$ & $\mathrm{b}_{2} \mathrm{C}_{\mathrm{j}}^{\mathrm{k}}$ & $\mathrm{b}_{3} \mathrm{C}_{\mathrm{j}}^{\mathrm{k}}$ & $\mathrm{b}_{4} \mathrm{C}_{\mathrm{j}}^{\mathrm{k}}$ & $\ldots$ & $\mathrm{b}_{\mathrm{n}} \mathrm{C}_{\mathrm{j}}^{\mathrm{k}}$ & \\
\hline $\mathrm{S}_{\mathrm{a}}$ & $\mathrm{S}_{\mathrm{p}}$ & $S_{n p}$ & $\mathrm{~S}_{\mathrm{np}}$ & & $\mathrm{S}_{\mathrm{p}}$ & \\
\hline $\mathrm{b}_{\mathrm{i}} \mathrm{C}_{\mathrm{j}}^{\mathrm{k}}$ & $\mathrm{b}_{2} \mathrm{C}_{\mathrm{j}}^{\mathrm{k}}$ & $\mathrm{b}_{3} \mathrm{C}_{\mathrm{j}}^{\mathrm{k}}$ & $\mathrm{b}_{4} \mathrm{C}_{\mathrm{j}}^{\mathrm{k}}$ & $\ldots$ & $\mathrm{b}_{\mathrm{n}} \mathrm{C}_{\mathrm{j}}^{\mathrm{k}}$ & $A b C_{j}^{g}$ \\
\hline - & 1 & 0 & 1 & $\ldots$ & 1 & Afinidade parcial \\
\hline
\end{tabular}

Figura 4. Comparação entre cadeia de padrões de antígeno e anticorpo com afinidade parcial.

\subsection{Critério de afinidade}

\section{A. Cura com afinidade total ou parcial}

A cura com afinidade total ou parcial entre anticorpo e antígeno somente poderá existir para células conhecidas. Neste caso, para uma anomalia na seção $k$ da célula $C_{j}$ tem-se o antígeno $\mathrm{AgC}_{\mathrm{j}}^{\mathrm{k}}$. Se existir um anticorpo $\mathrm{AbC}_{\mathrm{j}}^{\mathrm{k}}$ na memória do banco de dados, significa que este anticorpo é uma cura total para a anomalia. No entanto, caso não exista um anticorpo ligado ao antígeno na memória do banco de dados, a cura poderá ser encontrada pelo treinamento da rede elétrica através do algoritmo TS. Caso, o algoritmo TS não encontre um anticorpo com afinidade total ao antígeno, este poderá encontrar anticorpos com afinidade parcial ao antígeno. Assim, anticorpos com afinidade parcial ao antígeno existentes na memória do banco de dados, bem como encontrados no treinamento da rede pelo algoritmo TS, podem ser curas parciais para anomalias em células.

\section{B. Determinação de afinidade parcial}

$\mathrm{O}$ anticorpo com afinidade parcial ao antígeno poderá ser utilizado da seguinte forma:

- Como solução inicial do algoritmo TS: caso um anticorpo possua alguma afinidade parcial ao antígeno, sua cadeia de padrões poderá ser usada como solução inicial para o treinamento da rede através do algoritmo TS na busca por soluções de boa qualidade para o PRSDEE.

- Como solução final para o PRSDEE: caso não seja encontrado nenhum anticorpo com afinidade total ao antígeno, a cadeia de padrões do anticorpo com a maior afinidade parcial ao antígeno existente no banco de dados ou obtido através do algoritmo TS poderá ser usada como solução final para o PRSDEE.

No MGD-SIA, para avaliar a afinidade parcial entre anticorpo e antígeno, utiliza-se o conceito de casamento parcial e afinidade proposto em [9]. A taxa de afinidade representa o grau de semelhança necessária para ocorrer o casamento entre as duas cadeias em análise, definida como: 


$$
T_{A f}=\left(\frac{A_{n}}{A_{t}}\right) * 100
$$

sendo: TAf é a taxa de afinidade; $A_{n}$ é o número de cadeias normais no problema (cadeias próprias); At é o número total de cadeias no problema (cadeias próprias e não próprias).

A afinidade entre os dois padrões é dada por:

$$
A_{f t}=\frac{\sum_{i=1}^{L} P C}{L} * 100
$$

sendo: $A f t$ é a porcentagem de afinidade entre os padrões analisados; $L$ é a quantidade total de posições; $P C$ é a posição casada; $\sum_{i=1}^{L} P C$ é o somatório (quantidade) de posições casadas.

De acordo com o trabalho apresentado em [9], se $A f t$ for maior ou igual a $T A f(A f t \geq T A f)$ ocorre o casamento entre os dois padrões, ou seja, os dois são considerados semelhantes. Caso $A f t$ seja menor que TAf $(A f t<T A f)$ o anticorpo não reconhece o antígeno, portanto não existe casamento entre os padrões. No entanto, no PRSDEE, para haver algum grau de afinidade entre os padrões do anticorpo e antígeno, Aft deverá ser maior que TAf $(A f t>T A f)$. Neste caso, se $A f t$ for menor ou igual $T A f(A f t \leq T A f)$ não existe casamento parcial entre os padrões.

\section{Testes E Resultados}

Para testar a eficiência do MGD-SIA, apresentam-se resultados de testes para um defeito (anomalia) em um sistema de distribuição (célula) de média tensão, considerando que a célula é conhecida, ou seja, já foi analisada, porém com anomalia desconhecida, ou seja, não possui antígeno (defeito) e nem anticorpo com afinidade total (solução) na memória do banco de dados. Como a anomalia não possui anticorpos na memória do banco de dados, será necessário efetuar o treinamento da rede para a obtenção de anticorpos (soluções) com afinidade total ou parcial ao antígeno (defeito) da anomalia. Além disso, como a célula já foi analisada, possui anticorpos na memória do banco de dados que poderão servir como solução inicial (anticorpos com afinidade parcial ao antígeno) para o treinamento da rede através do algoritmo TS. A solução final para o PRSDEE deverá possuir uma cadeia de padrões de anticorpo com afinidade total ao antígeno, ou mesmo parcial que seja melhor que a solução inicial utilizada pelo algoritmo TS.

Considere então uma anomalia na seção 3 da célula $C_{53}$. Trata-se de uma célula existente no banco de dados, porém com antígeno desconhecido, contendo 53 seções (50 seções de carga e 3 seções representando as subestações). Os dados da célula $\mathrm{C}_{53}$ encontram-se em [8].

\section{A. Dados inicias}

O conjunto de células LC do banco de dados é dado por:

- $\mathrm{LC}=\left\{\mathrm{C}_{12}, \mathrm{C}_{53}, \mathrm{C}_{120}, \ldots, \mathrm{C}_{\mathrm{ns}}\right\}$
- $\mathrm{C}_{53}: \mathrm{S} 53$ (sistema de 53 seções)

Para a célula $\mathrm{C}_{53}$, temos o seguinte conjunto de antígenos:

- $\mathrm{LAgC}_{53}=\left\{\mathrm{AgC}_{53}^{2}, \mathrm{AgC}_{53}^{7}, \mathrm{AgC}_{53}^{8}, \mathrm{AgC}_{53}^{11}\right\}$

Para a anomalia na seção 3 da célula $\mathrm{C}_{53}$ temos:

- Antígeno da anomalia: $\mathrm{AgC}_{53}^{3}$

- $\mathrm{AgC}_{53}^{3} \notin \mathrm{LAgC}_{53}$

\section{B. Anomalia na célula}

A Fig. 5 ilustra a célula $\mathrm{C}_{53}$ (rede em operação). Considere então um defeito (anomalia) na seção 3 ( $\left(\mathrm{S}_{3} \mathrm{C}_{53}^{3}\right)$, e a Fig. 6 mostra a cadeia de padrões não próprios do antígeno $\left(\mathrm{AgC}_{53}^{3}\right)$ para esta anomalia. Com o defeito na seção 3, as seções 4, 5 , 6, 7, 8, 26, 27 e 28 ficaram desenergizadas. Para esta anomalia, a cadeia de padrões do antígeno $\mathrm{AgC}_{53}^{3}$ é composto pelas seções $\mathrm{S}_{\mathrm{a}}, \mathrm{S}_{\mathrm{p}}$ e $\mathrm{S}_{\mathrm{np}}$ :

- $\operatorname{AgC}_{53}^{3}\left(\mathrm{~S}_{\mathrm{a}}\right)=\left\{\mathrm{S}_{3} \mathrm{C}_{53}^{3}\right\}=\{3\}$

- $\mathrm{AgC}_{53}^{3}\left(\mathrm{~S}_{\mathrm{np}}\right)=\left\{\mathrm{S}_{4} \mathrm{C}_{53}^{3}, \mathrm{~S}_{5} \mathrm{C}_{53}^{3}, \mathrm{~S}_{6} \mathrm{C}_{53}^{3}, \mathrm{~S} \mathrm{C}_{53}^{3}, \mathrm{~S}_{53}^{3}\right.$, $\mathrm{S}_{26 \mathrm{C}_{53}^{3}}^{3}, \mathrm{~S}_{\left.27 \mathrm{C}_{53}^{3}, \mathrm{~S}_{2} 8 \mathrm{C}_{53}^{3}\right\}=\{4,5,6,7,8,26,27,28\}}$

- $\operatorname{AgC}_{53}^{3}\left(\mathrm{~S}_{\mathrm{p}}\right)=$ todas as demais 45 seções do sistema.

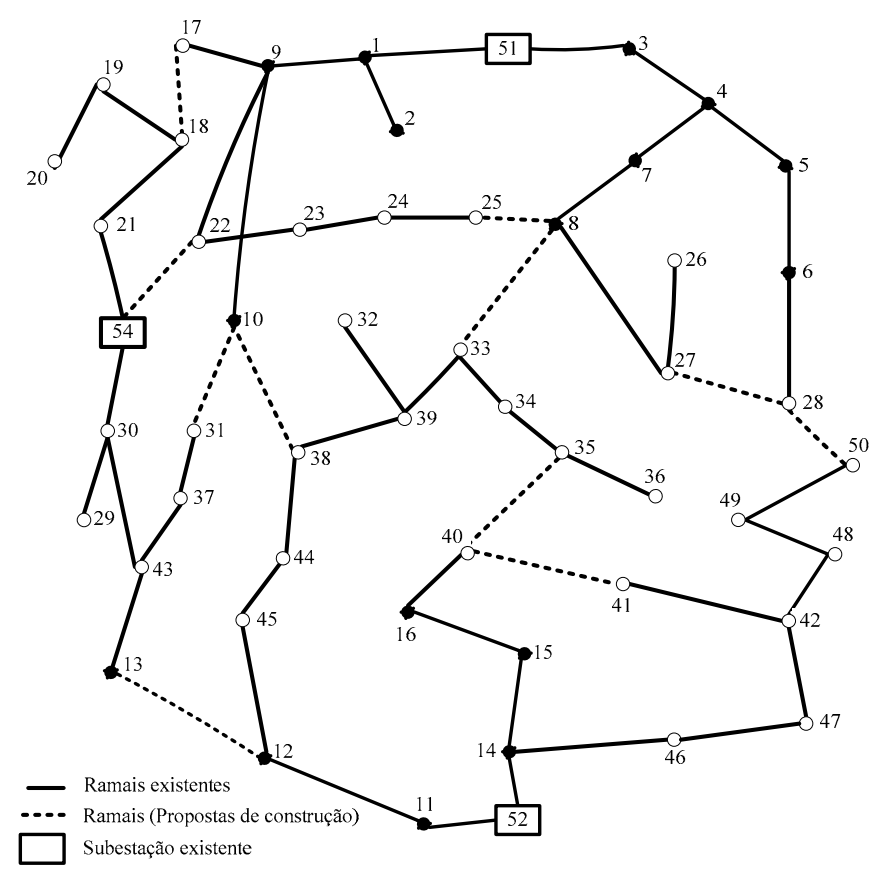

Figura 5. Célula $\mathrm{C}_{53}$ em operação antes de ocorrer o defeito na seção 3.

\begin{tabular}{|c|c|c|c|c|c|c|c|}
$\mathrm{S}_{\mathrm{np}}$ & $\mathrm{S}_{\mathrm{np}}$ & $\mathrm{S}_{\mathrm{np}}$ & $\mathrm{S}_{\mathrm{np}}$ & $\mathrm{S}_{\mathrm{np}}$ & $\mathrm{S}_{\mathrm{np}}$ & $\mathrm{S}_{\mathrm{np}}$ & $\mathrm{S}_{\mathrm{np}}$ \\
\hline 4 & 5 & 6 & 7 & 8 & 26 & 27 & 28 \\
\hline
\end{tabular}

Figura 6. Cadeia de padrões não próprios $\left(\mathrm{S}_{\mathrm{np}}\right)$ do antígeno $\mathrm{AgC}_{53}^{3}$.

\section{Solução inicial do treinamento}

Após checar a lista de antígenos da célula $\mathrm{C}_{53}$, o MGD-SIA encontrou $\mathrm{o}$ anticorpo $\mathrm{AbC}_{53}^{7}$ com afinidade parcial ao antígeno $\mathrm{AgC}_{53}^{3}$. Este anticorpo será usado para iniciar o algoritmo TS. Trata-se de um anticorpo gerado para a anomalia na seção 7 da célula $C_{53}$. Para esta anomalia, a 
cadeia de padrões do antígeno $\mathrm{AgC}_{53}^{7}$ (Fig. 7) é composto pelas seções $\mathrm{S}_{\mathrm{a}}, \mathrm{S}_{\mathrm{p}}$ e $\mathrm{S}_{\mathrm{np}}$ :

- $\operatorname{AgC}_{53}^{7}\left(\mathrm{~S}_{\mathrm{a}}\right)=\{7\}$

- $\operatorname{AgC}_{53}^{7}\left(\mathrm{~S}_{\mathrm{np}}\right)=\{8,26,27\}$

- $\operatorname{AgC}_{53}^{7}\left(\mathrm{~S}_{\mathrm{p}}\right)=$ todas as demais 50 seções do sistema.

$$
\begin{array}{|l|l|l|}
\hline \multicolumn{1}{|c|}{\mathrm{S}_{\mathrm{np}}} & \mathrm{S}_{\mathrm{np}} & \mathrm{S}_{\mathrm{np}} \\
\hline 8 & 26 & 27 \\
\hline
\end{array}
$$

Figura 7. Cadeia de padrões não próprios do antígeno $\mathrm{AgC}_{53}^{7}$.

A afinidade parcial entre o anticorpo $\mathrm{AbC}_{53}^{7}$ e o antígeno $\mathrm{AgC}_{53}^{3}$ foi checada através das equações 1 e 2 , sendo:

$T_{A f}=\left(\frac{45}{53}\right) * 100=84,9 \%$

$A_{f t}=\frac{50}{53} * 100=94,3 \%$

Neste caso temos que $A f t>T A f$. Portanto existe afinidade parcial entre o anticorpo $\mathrm{AbC}_{53}^{7}$ e o antígeno $\mathrm{AgC}_{53}^{3}$. Assim, este anticorpo será usado como solução inicial para o treinamento da rede através do algoritmo TS.

\section{Cura para a célula}

Após treinar a rede através do algoritmo TS, a cura encontrada para a anomalia na seção 3 da célula $C_{53}$, é dada então pelo anticorpo $\mathrm{AbC}_{53}^{3}$. A Fig. 8 ilustra a célula $\mathrm{C}_{53}$ (sistema em operação) após a cura para a anomalia na seção 3 (anticorpo $\mathrm{AbC}_{53}^{3}$ ).

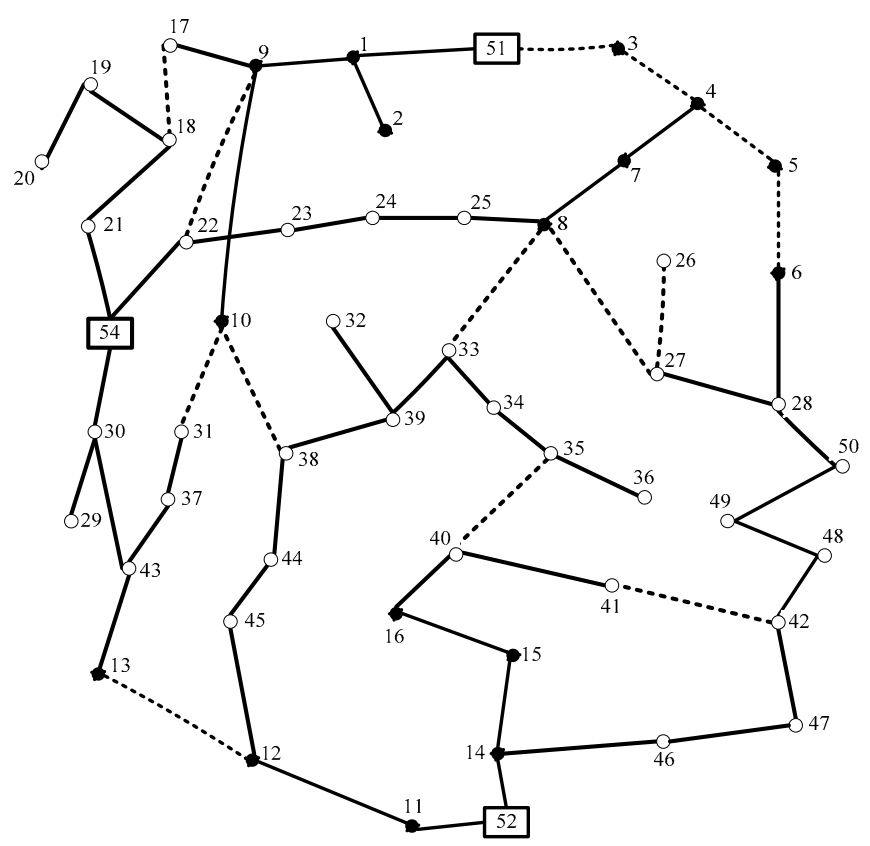

Figura 8. Cura para a anomalia na seção 3 da célula $C_{53}$.

Para o antígeno $\mathrm{AgC}_{53}^{3}$, temos a seguinte cadeia de padrões do anticorpo $\mathrm{AbC}_{53}^{31}$ :
- $\mathrm{AbC}_{53}^{3}=\{4,0,6,7,8,0,27,28\}$

A Fig. 9 ilustra a afinidade entre os padrões do antígeno $\mathrm{AgC}_{53}^{3}$ e anticorpo $\mathrm{AbC}_{53}^{31}$.

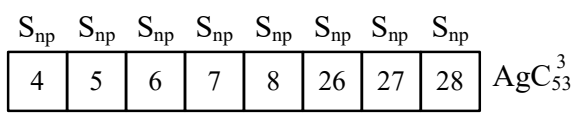

\begin{tabular}{|l|l|l|l|l|l|l|l|l|}
\hline 4 & 0 & 6 & 7 & 8 & 0 & 27 & 28 & $\mathrm{AbC}_{53}^{3}$ \\
\hline
\end{tabular}

\begin{tabular}{|l|l|l|l|l|l|l|l|}
\hline 1 & 0 & 1 & 1 & 1 & 0 & 1 & 1 \\
Afinidade parcial
\end{tabular}

Figura 9. Afinidade parcial ente o antígeno $\mathrm{AgC}_{53}^{3}$ e anticorpo $\mathrm{AbC}_{53}^{3}$.

De acordo com a Fig. 9, percebe-se que além da seção onde ocorreu o defeito, as seções 5 e 26 não foram restauradas pelo algoritmo TS, pois violou uma das restrições impostas ao problema de restauração. A solução encontrada conseguiu restaurar as seções 4, 6, 7, 8, 27 e 28 . Neste caso, tem-se uma solução com um anticorpo com afinidade parcial ao antígeno.

O tempo de processamento de treinamento da rede através do algoritmo TS partindo-se de uma solução inicial conhecida foi de 0,257 segundos. A mesma solução encontrada pelo algoritmo TS sem utilizar uma solução inicial de boa qualidade, obteve um tempo de processamento de 0,343 segundos.

\section{CONCLUSÕES}

De acordo com os resultados, a ferramenta desenvolvida foi capaz de gerenciar as soluções (solução inicial existente e solução final) entre o banco de dados e o treinamento de rede. Além disso, o fato de utilizar uma solução inicial existente no banco de dados no treinamento da rede para encontrar uma solução final de boa qualidade reduziu o tempo de processamento. No entanto, este fato deve ser melhor visto em sistemas de grande porte.

Para anomalias em células que nunca foram analisadas, não existem dados na memória do banco de dados referente a essa célula. Neste caso, o algoritmo MGD-SIA deverá identificar que a célula é desconhecida e treinar a rede através do algoritmo TS para tentar resolver o problema de PRSDEE. Os resultados serão armazenados no banco de dados e poderão ser utilizados na resolução desta ou de outras anomalias na mesma célula. No entanto, para anomalias em células já analisadas e com antígenos conhecidos, o algoritmo MGDSIA identificará que a célula e o antígeno são conhecidos e encontrará o anticorpo (solução) com afinidade ao antígeno no banco de dados. Neste caso, o tempo de resposta na busca de soluções para o PRSDEE será rápido.

$\mathrm{O}$ algoritmo MGD-SIA pode ser utilizado para que o operador do sistema elétrico treine situações de defeito em toda a rede e armazene os dados das soluções na memória do banco de dados para que possam ser usados para resolver problemas futuros. Ressalta-se, que o método de gerenciamento e memória de dados desenvolvido para o PRSDEE através da abordagem imunológica é simples e eficiente, e que pode ser adaptado para ser utilizado para 
outras ferramentas com aplicações diversas na área de sistemas elétricos de potência.

\section{REFERÊNCIAS}

[1] L. N. Castro, "Immune engineering: development and application of computational tools inspired by artificial immune systems," $P h D$ thesis, UNICAMP, 2001.

[2] D. Dasgupta, "Artificial immune systems and their applications", Springer, 1998.

[3] S. Toune, H. Fudo, T. Genji, Y. Fukuyama and Y. Nakanishi, "Comparative study of modern heuristic algorithms to service restoration in distribution systems," IEEE Transactions on Power Delivery, vol. 17, pp. 173-181, 2002.

[4] B. Akduman, B. Turkay and A. S. Uyar, "Service restoration in distribution systems using an evolutionary algorithm," Power Generation, Transmission, Distribution and Energy Conversion, 2010.

[5] D. S. Sanches, J. B. A. London Junior and A. C. B. Delbem, "Multiobjective evolutionary algorithm for single and multiple fault service restoration in large-scale distribution systems," Electric Power Systems Research, vol. 110, pp. 144-153, 2014.

[6] A. Y. Abdelaziz, F. M. Mohamed, S. F. M. A. L. Mekhamer and M. A. L. Badr "Distribution system reconfiguration using a modified Tabu Search algorithm," Electric Power System Research, vol. 80, pp. 943953, 2010.

[7] F. R. Alonso, D. Q. Oliveira, A. C. and Zambroni de Souza "Artificial Immune Systems Optimization Approach for Multiobjective Distribution System Reconfiguration," IEEE Transactions on Power Systems, vol. 30, pp. 840-847, 2015.

[8] V. Miranda, J. V. Ranito and L. M. Proença, "Genetic Algorithms in Optimal Multistage Distribution Network Planning," IEEE Transactions on Power Systems, vol. 9, no. 4, pp. 1927-1933, 1994.

[9] D. W. Bradley and A. M. Tyrrell, "Immunotronics - NovelFinite-StateMachine Architectures with Built-InSelf-Test Using Self-Nonself Differentiation," IEEE Transactions on Evolutionary Computation, vol. 6, pp. 227-238, 2002.

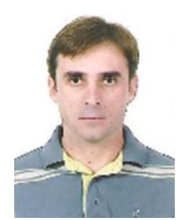

Antonio Marcos Cossi é, graduado em Engenharia Elétrica pela Universidade Estadual Paulista "Júlio de Mesquita Filho" (UNESP), Illha Solteira, São Paulo, Brasil, em 2000. Obteve o título de Mestre e Doutor em Engenharia Elétrica pela Universidade Estadual Paulista "Júlio de Mesquita Filho" (UNESP), Ilha Solteira, São Paulo, Brasil, em 2000 e 2008 , respectivamente. Atualmente é professor e pesquisador no Departamento de Matemática da Universidade Estadual Paulista "Júlio de Mesquita Filho" (UNESP), Ilha Solteira, São Paulo, Brasil. Suas pesquisas se concentram nas áreas de Planejamento e Controle de Sistemas de Energia Elétrica, atuando principalmente nos seguintes temas: Planejamento, restauração, confiabilidade e qualidade do fornecimento de energia elétrica, de sistemas de distribuição de energia elétrica.

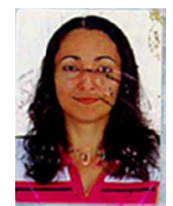

Mara Lúcia Martins Lopes é, graduada em Licenciatura Plena pela Universidade Federal de Mato Grosso do Sul (UFMS), Três Lagoas, Mato Grosso do Sul, em 1997. Obteve o título de Mestre e Doutora em Engenharia Elétrica pela Universidade Estadual Paulista "Júlio de Mesquita Filho" (UNESP), Ilha Solteira, São Paulo, em 2000 e 2005, respectivamente. Possui Pós-Doutorado em Engenharia pela Universidade Estadual Paulista "Júlio de Mesquita Filho" (UNESP), Ilha Solteira, São Paulo, obtido em 2007. Atualmente é professora e pesquisador no Departamento de Matemática da Universidade Estadual Paulista "Júlio de Mesquita Filho" (UNESP), Ilha Solteira, São Paulo, Brasil. Suas pesquisas se concentram nas áreas de Bioestatística, Engenharia Elétrica, com ênfase em Transmissão e Distribuição de Energia Elétrica, atuando principalmente nos seguintes temas: redes neurais artificiais (back-propagation, teoria da ressonância adaptativa, outros), lógica nebulosa, previsão de cargas elétricas, aproximação de funções. 